\title{
Topics
}

\section{Review of Pulsed Plasma Thruster Development at IRS}

\author{
By Tony SCHÖNHERR ${ }^{1)}$, Anuscheh NAWAZ ${ }^{2,3)}$, Matthias LAU ${ }^{3)}$, Dejan PETKOW ${ }^{3)}$ and Georg HERDRICH ${ }^{3)}$ \\ ${ }^{1)}$ Department of Advanced Energy, The University of Tokyo, Kashiwa, Japan \\ ${ }^{2)}$ now at: Sierra Lobo Inc. contractor at NASA Ames Research Center, Moffett Field, CA, USA \\ ${ }^{3)}$ Institute of Space Systems (IRS), Universität Stuttgart, Stuttgart, Germany
}

(Received June 15th, 2009)

\begin{abstract}
The research and development process of the ablative pulsed plasma thruster SIMP-LEX is summarized. By experiment, the magnetic field in between the electrodes was measured with an induction probe, and a high-speed camera was applied to estimate the propagation of the plasma plume. Further, the optimization process towards higher thrust efficiency by means of geometric and electrical modifications is described. By analysis, the calculation of magnetic field and change in inductance is summarized and the numerical models for the performance simulation of SIMP-LEX introduced. A peak value of $0.7 \mathrm{~T}$ was found for the magnetic field created during an $80 \mathrm{~J}$ discharge whereas a propagation velocity of $28 \mathrm{~km} / \mathrm{s}$ was estimated for the first plasma plume. The optimization process towards a new engineering model yielded an increase of $40 \%$ in mean exhaust velocity to $26 \mathrm{~km} / \mathrm{s}$, and a maximum thrust efficiency of $32 \%$ was evaluated respectively. Calculation of magnetic field and change in inductance based on the Biot-Savart law yield input for the slug model, which can then predict the general performance parameters with a good accordance.
\end{abstract}

Key Words: Pulsed Plasma Thruster, Electric Propulsion, Thruster Development

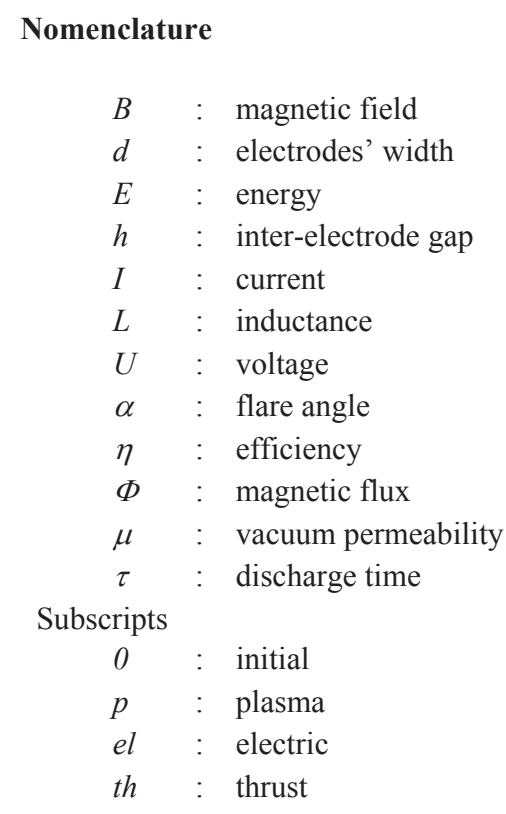

\section{Introduction}

The Institute of Space Systems (IRS) is undergoing the challenge of planning, designing, studying and assembling of several small satellites within the Stuttgart Small Satellite Program $^{1)}$. As part of this endeavor, the mission Perseus will be used, inter alia, as a test bed for the electric propulsion systems developed at the IRS. Scheduled to be placed in a low Earth orbit (LEO), the satellite is intended to have a mass of less than $150 \mathrm{~kg}$ comprising one arcjet thruster and one pulsed plasma thruster (PPT). After the in-orbit validation of the performance characteristics and the operational behavior, the subsequently refined thrusters will be applied to the Lunar Mission $B W 1^{2)}$.

As a first step of the development of the PPT, an engineering model was set up and first operational tests and scientific investigations were launched ${ }^{2,3)}$. Concurrently, the modeling of the thruster was envisaged in order to compare with experimental results as well as to obtain a better understanding of the related physical theories.

A review of the experimental and the theoretical studies concerning the development of the PPT named SIMP-LEX (Stuttgart Impulsing Magnetoplasmadynamic Thruster for Lunar Exploration) is presented in the following chapters.

As a result of these experimental studies, and with support of RIAME MAI (Research Institute of Applied Mechanics and Electrodynamics of Moscow Aviation Institute), a new improved thruster design could be acquired named ADD SIMP-LEX (ADvanceD SIMP-LEX) ${ }^{3)}$.

\section{Experimental Investigations}

Following the studies subsequent to the setup of the thruster facility, experimental investigations were conducted to obtain information about the operational behavior and the performance characteristics of the PPT. The equipment used therefore comprises vacuum chambers with pertinent pumping systems, a highly precise mass balance, a thrust balance, a high-speed camera as well as magnetic field probes ${ }^{4,5}$.

Additionally, studies were undertaken to optimize the thruster with respect to the mission's requirements, i.e., the mean exhaust velocity and the thrust efficiency. A geometric as well as an electrical parameter study approach was used. For the evaluation of the parametric results, the statistical method using Taguchi matrices was used. 
The PPT SIMP-LEX, which was used in the experiments, is a pulsed magnetoplasmadynamic thruster of about $40 \times 25 \times$ $10 \mathrm{~cm}^{3}$ consisting of four capacitors with a total capacitance of $35 \mu \mathrm{F}^{6)}$.

The capacitor voltage varies between 1500 and $2000 \mathrm{~V}$ so that the total energy of the capacitor bank has a range of 40 to $70 \mathrm{~J}$. The propellant used for all experiments is PTFE (polytetrafluoroethylene). In Figure 1, the thruster SIMP-LEX is shown.

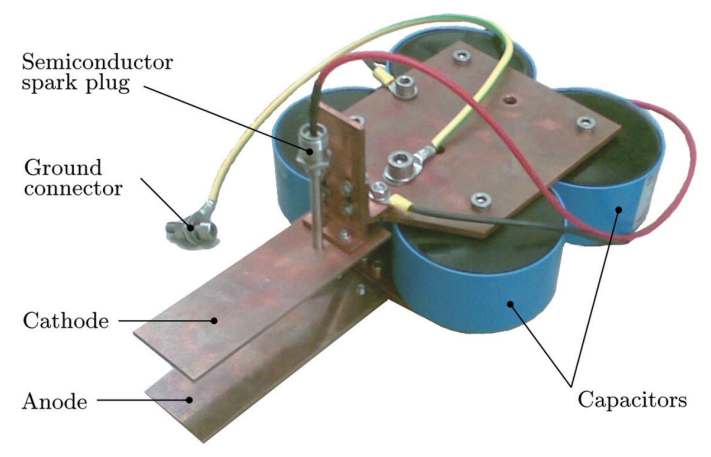

Fig. 1. Thruster setup of SIMP-LEX.

\subsection{Magnetic field measurements}

The thruster's self-induced magnetic field $B$ has been investigated by means of intrusive induction probes ${ }^{3,7)}$. An induction probe delivers a voltage signal according to the Faraday law when exposed to a time varying magnetic field. The method has been successfully employed by a number of researchers in the past and has shown to yield trustful results ${ }^{8}$ 9). Recording the magnetic field facilitates thruster optimization, understanding of the acceleration process, estimation of the acceleration force and evaluation of the $B$-field model. The analytical model of the magnetic field was modified for this investigation by introducing a current sheet thickness. The study at IRS included measurement of the spatial and temporal magnetic field inside the acceleration channel of the SIMP-LEX thruster head. A specially shielded induction probe was built, calibrated and used to access the magnetic field along predefined points on a measurement axis and in the propellant exit-plane. The voltage signals were computed into the magnetic field, by using a Helmholtz coil for calibration, since the probes voltage response depends on the frequency spectrum of the time varying magnetic field.

The probes induction coil has a diameter of $1 \mathrm{~mm}$ and a length of $3.5 \mathrm{~mm}$ and is made of 15 turns of insulated copper wire with a special layout to prevent unwanted coupling effects. It is further shielded by layers of aluminum foil and a glass tube. The setup provides good spatial resolution and showed excellent response. The upper limit for the frequency calibration was set to $300 \mathrm{kHz}$ after evaluating the discharge frequency spectrum of SIMP-LEX. The probe was mounted on a two-axis table to remote control its position between the electrodes during trials inside the vacuum chamber. The thruster setup was changed between a side-fed and a breech-fed configuration of the solid PTFE propellant to investigate the influence. Further, the recorded magnetic fields were analyzed, related to high-speed-camera (HSC) pictures and compared to the model predictions. Benchmark

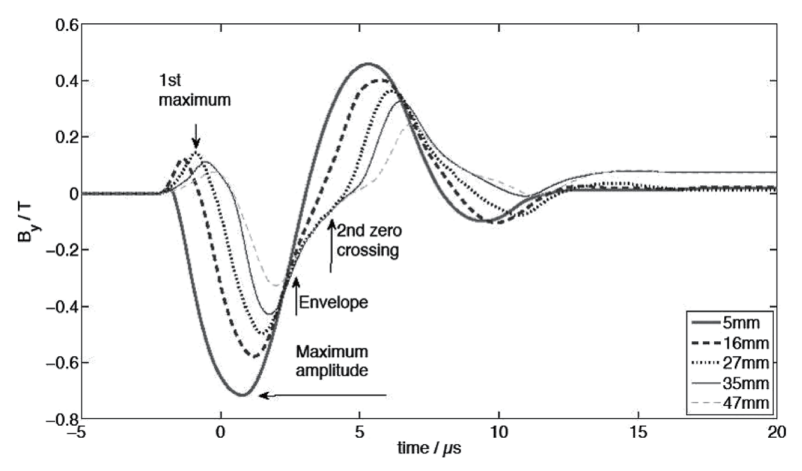

Fig. 2. Magnetic field measurement between the electrodes of SIMP-LEX for $U_{0}=2000 \mathrm{~V}$ and at different distances $(5-47 \mathrm{~mm})$ of the probe from the propellant exit plane along the electrode axis, breech-fed ${ }^{7}$.

measurement results for a breech-fed configuration for different positions of the probe can be found in Figure 2.

The measured magnetic field showed an overall peak at 0.7 $\mathrm{T}$ and stayed within prediction boundaries according to the thruster's energy level. The recorded magnetic field distributions agreed very well with literature and showed clear distinctions between breech-fed and side-fed propellant configurations. It is interesting to note, that an induced current loop interaction described prior to this work by Palumbo and Begun ${ }^{9)}$ could be verified experimentally in this study. Effects of the current sheet thickness on the magnetic field distribution were found both, by experiments and modeling. The comparison of the predicted and the measured magnetic field at two positions only showed good accordance at the PTFE surface position for the first $10 \mu$ s of the discharge. The simulations revealed the strong correlation between discharge current and magnetic field to improve future results. From the measurements a current sheet velocity of $20 \mathrm{~km} / \mathrm{s}$ was derived for the breech-fed configuration. However, this was not possible for the side-fed configuration due to an interference effect.

\subsection{High-speed camera measurements}

To aid in the development of the discharge model for SIMP-LEX the plasma sheet propagation was investigated by means of a high speed camera sensitive to wavelengths between $380-900 \mathrm{~nm}^{3,5}$. The plasma sheet was analyzed to determine the position as a function of time, form and overall discharge behavior. This information was correlated to simulations, literature data and other experiments on SIMP-LEX. The plasma sheet only remains between the electrodes for the very short time of several microseconds. To monitor the sheet with sufficient temporal resolution pictures were taken every $100 \mathrm{~ns}$. The camera used is capable of taking one picture per discharge, so the pictures of multiple discharges were combined to a series manually to get the overall propagation. Thus a complicated trigger setup provided exact timing of the discharge initiation and camera activation with respect to a reproducible time reference.

Pictures were taken for three different electrode distances and capacitor voltages. From the pictures it was possible to gain information about three clearly discernible phases of one discharge pulse each featuring an own plasma front. Velocities 
of the first plasma front were found to be in the $28 \mathrm{~km} / \mathrm{s}$ region for a capacitor voltage of $2000 \mathrm{~V}$ and a gap of $36 \mathrm{~mm}$ between the electrodes. The canting of a plasma front as well as the non-uniform brightness distribution of the front makes it impossible to determine an exact location of the actual plasma sheet as can be seen for the pictures shown in Figure 3.

However, the change in position and velocity was determined. This was achieved by a MatLab-algorithm developed identifying the plasma sheet edge during picture post processing.
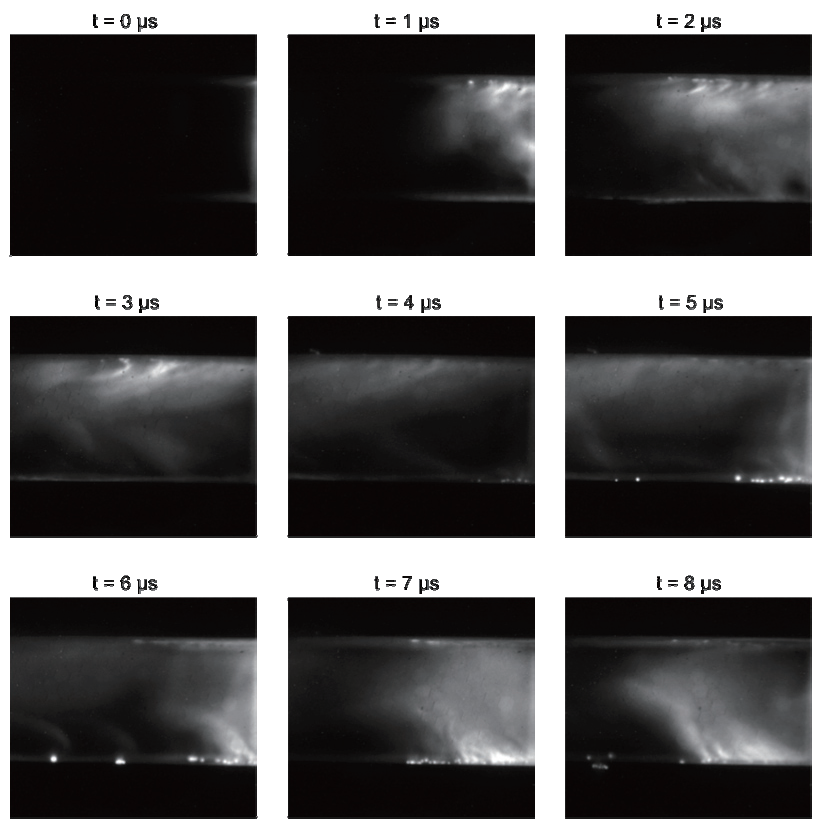

Fig. 3. High-speed camera pictures of SIMP-LEX for $U_{0}=2000 \mathrm{~V}^{5)}$.

\subsection{Geometric design optimization}

Optimum performance of the PPT increases the payload capacity of the satellite and is, thus, a highly interesting aim.

Several variations in the electrode's geometry were investigated for this purpose ${ }^{3,10)}$. Based on other findings ${ }^{11)}$, these variations include the length and width of the electrodes as well as the gap between them, and the applied capacitor voltage $^{4,6)}$. Further, the electrodes were changed in shape, i.e., flared and tongue shaped electrodes were used with expectations of an increase in performance. Systematic parameter changes were conducted by direct parametric permutations as well as with help of the so-called Taguchi method $^{12)}$. The variations made during the parametric investigation are summarized in Tab. 1.

The bank energy $E_{0}$ was changed by means of the capacitor voltage $U_{0}$. The electrodes' width $d$ was changed as well as the distance between the electrode plates $h$.

Table 1. Investigated parameters for optimization of SIMP-LEX ${ }^{4}$.

\begin{tabular}{|l|l|}
\hline Voltage, $\mathrm{V}$ & $1500,1800,2000$ \\
\hline Electrode width, $\mathrm{mm}$ & $20,40,60$ \\
\hline Distance between electrodes, $\mathrm{mm}$ & $21,36,42$ \\
\hline Length of electrodes, $\%$ & 75,100 \\
\hline Flare angle, deg & $0,10,20,30$ \\
\hline Tongue shape & yes, no \\
\hline
\end{tabular}

The length of the electrodes was varied in order to investigate the influence on the exhaust velocity. In addition, an increase in change in inductance $\Delta L$ was enforced by flaring the electrodes by an angle of $\alpha$ as well as using a tongue shape.

The direct parametric permutations were used to identify main influences of parameters, while the Taguchi method could also be used to investigate the interactions between the parameters and their influences on the target values being the impulse bit, the mass bit, the exhaust velocity and the thrust efficiency.

The results found ${ }^{4,6)}$ showed major tendencies that yielded conclusions about a subsequent model. In general, a decrease in mean exhaust velocity is noticed with greater values of $h$. For higher energies, however, the exhaust velocity decreases less, and can even turn into an increase. This could also be validated with the Taguchi matrix. As for the electrode's geometry a maximum in exhaust velocity was found for a minimum in width and length. The flare angle showed a positive effect on the overall thruster performance for an angle up to about $20 \mathrm{deg}$. The change in shape towards a tongue also raised the mean exhaust velocity and the thrust efficiency.

Combining both effects of flared and tongue-shaped electrodes yielded an increase in thrust efficiency by nearly $35 \%$ and in exhaust velocity of nearly $20 \%{ }^{6}$.

An optimum configuration for the thruster's electrodes was concluded regarding the highest mean exhaust velocity of the plasma plume and the highest thrust efficiency ${ }^{4,6}$. . Thus, respecting the Tsiolkovsky equation, the propellant needed is reduced and the satellite may be equipped with more scientific hardware.

\subsection{Electrical circuit design optimization}

Not only the above-mentioned geometrical aspects of the electrodes have an influence on the performance. Additionally, the parameters of the electrical oscillation circuit, i.e., the capacitance, the resistance and the inductance, have a strong effect on the discharge behavior, and, hence, the plasma creation and acceleration.

Especially it could be shown analytically that the thrust efficiency is a function of the characteristic discharge time $\tau_{c}$ as well as the basic inductance $L_{0}$ of the circuit ${ }^{3,13)}$.

In order to investigate these effects and based on the results of the geometric optimization, a newly designed thruster engineering model named ADD SIMP-LEX was introduced, as can be seen in Figure 4.

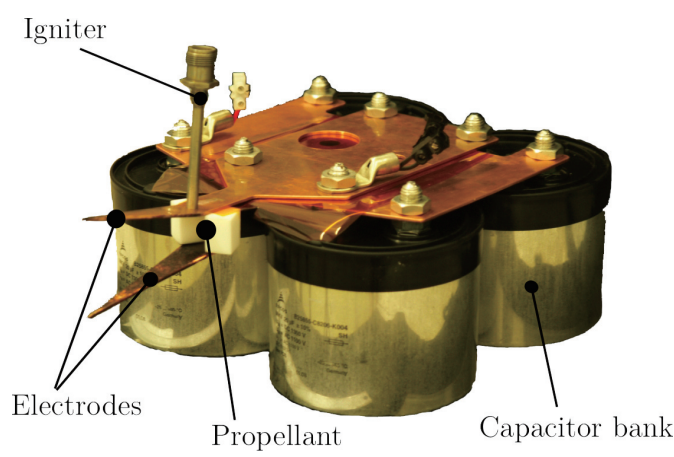

Fig. 4. Thruster setup of ADD SIMP-LEX ${ }^{3,13)}$ 
Both thruster models have significantly different circuit parameters. Measuring the voltage yields information about the mean resistance and inductance during the discharge, neglecting the time-dependent influence of the plasma. Summarized in Table 2 are the parameters for both setups.

Table 2. Electrical circuit parameters of the different thrusters ${ }^{13)}$.

\begin{tabular}{|l|c|c|}
\hline Thruster type & SIMP-LEX & ADD SIMP-LEX \\
\hline Bank energy, $\mathrm{J}$ & 68 & 68 \\
\hline Capacitance, $\mu \mathrm{F}$ & 34 & 80 \\
\hline Mean inductance, $\mathrm{nH}$ & 58.9 & 38.9 \\
\hline Mean resistance, $\mathrm{m} \Omega$ & 11.5 & 24.6 \\
\hline
\end{tabular}

Several experiments were conducted to investigate the influence of the design change and of a variation in capacitance for ADD SIMP-LEX.

While a decrease in ablated mass of $12 \%$ could be observed, the impulse bit stayed nearly constant for the same bank energy, yielding an increase in exhaust velocity and thrust efficiency for the new design. Further, it could be investigated that an optimum performance of ADD SIMP-LEX could be achieved at a capacitance of $60 \mu \mathrm{F}$, i.e., using only three of the initial four capacitors ${ }^{13)}$.

The key parameters of selected thruster configurations are presented in Table 3. It should be noted that the mass of the engineering models does not include the envisaged propellant for the satellite mission nor the propellant feed system.

Table 3. Key specifications of the different thrusters ${ }^{13)}$

\begin{tabular}{|l|c|c|c|}
\hline Thruster type & SIMP-LEX & ADDS & ADDS \\
\hline Bank energy, $\mathrm{J}$ & 68 & 16.7 & 68 \\
\hline Capacitance, $\mu \mathrm{F}$ & 34 & 60 & 80 \\
\hline Dry mass, $\mathrm{kg}$ & 4.5 & 5.2 & 6.7 \\
\hline Impulse bit, $\mu \mathrm{Ns}$ & 900 & 420 & 1375 \\
\hline Mass shot, $\mu \mathrm{g}$ & 50 & 16 & 50 \\
\hline Mean exh. velocity, $\mathrm{km} / \mathrm{s}$ & 18 & 26 & 26 \\
\hline Thrust efficiency, $\%$ & 12 & 32 & 26 \\
\hline
\end{tabular}

A history of the development can be found in Fig. 5 for the base engineering model of SIMP-LEX (SLP), the geometrically optimized SIMP-LEX (SLT) and the then electrically optimized ADD SIMP-LEX (ADDS). The thrust efficiency was compared and an increase between the first and the currently last thrusters of $100 \%$ (from $12 \%$ to $26 \%$ ) can be observed, and validates the success of the optimization.

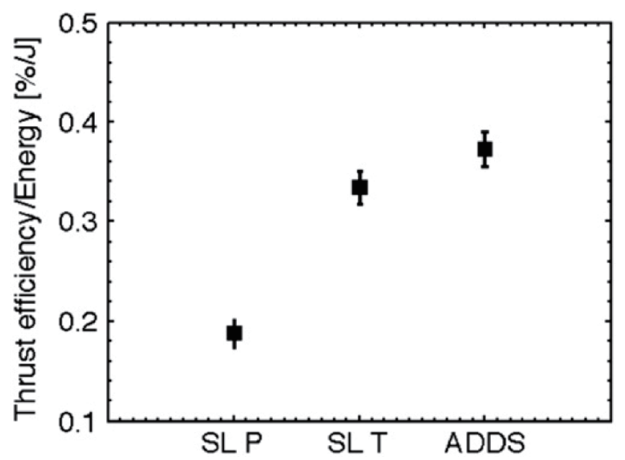

Fig. 5. Summary of development results for the thruster family ${ }^{13)}$

\section{Analytical and Numerical Studies}

To verify and to understand the experimental results, several different analyses were conducted. As the magnetic field plays an important role in the acceleration process of the plasma, effort was put into modeling the induced magnetic field. By these results it is also possible to simulate the behavior of the plasma, and, hence, the thruster. Although there are several different models proposed ${ }^{14)}$, only two models were more intensively considered for the description of SIMP-LEX ${ }^{3}$. One is based on the so-called slug model, and solves the basic differential equations for an entire plasma sheet whereas the much more complex model is based on a particle approach.

\subsection{Calculation of magnetic field and change in inductance}

Calculating the magnetic field $B$ between the electrodes can provide information not only about strength and shape of the magnetic field, but also leads to an estimation of the change in inductance $\Delta L$ in the electrical circuit caused by the plasma:

$$
\Delta L=\frac{\Phi}{I}=\frac{1}{I} \iint_{A} B \mathrm{~d} z \mathrm{~d} x
$$

where $I$ is the electrical current, $\Phi$ the magnetic flux, and $x$ and $z$ the axis along the length of the electrodes, along the gap respectively. The knowledge of this value is important for modeling the thruster, but also as a design criterion ${ }^{4)}$.

There are several methods to derivate the magnetic field, and, hence, the change in inductance from the electrical and geometric properties of the thruster, different in the assumptions that are made. For a simple estimation of the change in inductance ${ }^{14)}$, the edge effects occurring at the electrodes may be neglected, i.e., a homogeneous magnetic field in the cross section is assumed which is very distant from reality in SIMP-LEX due to the more complex electrodes' geometry that were investigated with SIMP-LEX as seen in Fig. 6 and 7.

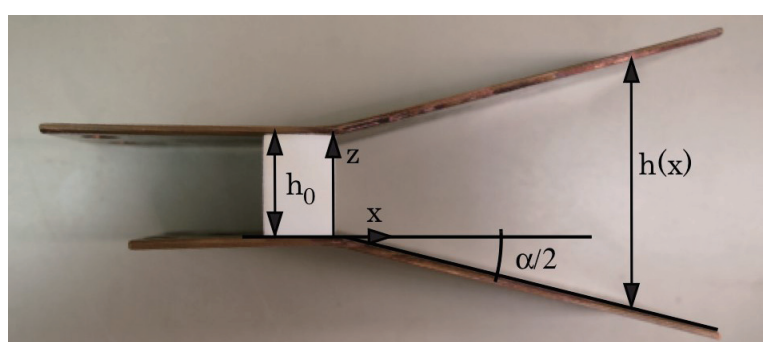

Fig. 6. Flared electrode geometry of SIMP-LEX ${ }^{3,6)}$.

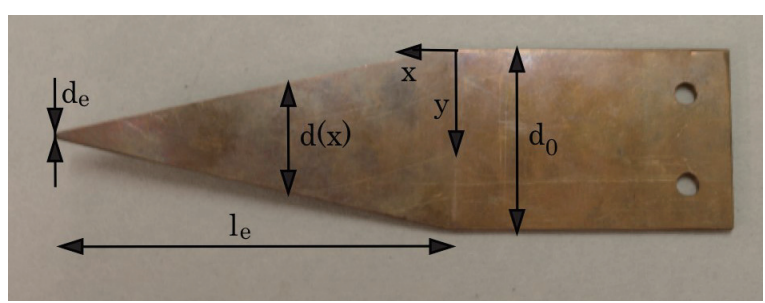

Fig. 7. Tongue-shaped electrode geometry of SIMP-LEX ${ }^{3,6)}$. 
However, the estimation can be used to have a rough and quick value calculated by ${ }^{14)}$ :

$$
\Delta L=\mu_{0} \int_{0}^{x_{p}} \frac{h(x)}{d(x)} \mathrm{d} x
$$

with $x_{p}$ being the current position of the plasma, as a current is only able to traverse the space between the electrodes via charged particles.

For a more detailed calculation, the Biot-Savart law can be applied $^{3,6,10,14)}$. The analysis method is basing on the fact that a number of finite current filaments is assumed each carrying a fraction of the total current, and the magnetic field induced is calculated normalized by the current ${ }^{5-7)}$.

Integrating over the space between the electrodes eventually leads to the change in inductance as a function of the plasma position, necessary for the modeling of the thruster's performance. This method was applied to several different geometries of the electrodes, and, thus, provides a tool to quantify effects of various changes in geometric values, e.g., the flare angle, and the above mentioned tongue shape (see $\mathrm{Ch}$. 2.3) respectively ${ }^{6}$. Calculations showed that an increase in flare angle and a decrease in aspect ratio, i.e., a tongue-shaped electrode yield an increase in change in inductance ${ }^{4,6}$.

The change in inductance is a sign for the quality of the electric circuit. The electrical efficiency $\eta_{\mathrm{el}}$ is found by ${ }^{4,14)}$ :

$$
\eta_{e l}<\frac{\Delta L}{L_{0}}
$$

That means, that an increase in change in inductance due to geometric changes of the electrodes (Ch. 2.3) and a decrease in the initial inductance due to an optimization of the thruster's circuit (Ch. 2.4) eventually yields a higher electric efficiency, and a, hence, higher thrust efficiency.

The increase in this efficiency found as a result of the experiments could be verified by the calculation method with the Biot-Savart law. However, the drop in performance beyond a flare angle of 20 deg could not be explained and, therefore, a model for the thruster's processes had to be taken into account. This model is explained in the following chapter.

\subsection{Slug model}

The model herein, called slug model ${ }^{14)}$, is aimed towards not only helpful in understanding the pulsed plasma thruster but also as a tool for designing future thrusters for planned missions. The focus is, hence, put to keep the model simple enough to being easily calculated, but complex enough to attain a certain accuracy. The slug model describes the acceleration of the plasma sheet within a PPT making some assumptions, e.g., that the mass ablated is accelerated in a plasma sheet without any variations in mass ${ }^{5)}$. In addition, the inductance is linear function of the plasma position.

By using the Biot-Savart approach outlined in the previous section to determine the inductance and merging it with the conventional slug model leads to a solution describing the movement of the plasma sheet. By that, the impulse bit as well as the exhaust velocities for every plasma sheet can be calculated. Comparison with the experimental results showed good accordance, albeit slight discrepancies could be observed $^{3,6,10)}$.

\subsection{Fully kinetic plasma model}

In order to investigate the processes in the low-density plasma more detailed than could be done with the slug model, the endeavor of creating a numerical code for the PPT was undertaken ${ }^{15}$. Facing the problems in describing a PPT, e.g., the low-density plasma, the chemical and thermal non-equilibrium in it, the effects of the electric and magnetic field, the spatially and temporarily inhomogeneous plasma, the complex ablation processes and so forth, a realistic model requires very profound knowledge of the plasma physics and a well-thought-out numerical code.

In cooperation with the Institute of Aerodynamics and Gasdynamics (IAG) of the Universität Stuttgart, the High Performance Computing Center Stuttgart (HLRS) and the Research Center in Karlsruhe (FZK), the IRS develops a 3D particle code for the fully kinetic simulation of pulsed plasma thrusters.

For the description of the non-continuum plasma, the solution of the full Boltzmann equation is approximated by combining three methods which are usually applied for different plasma and gas dynamic problems. Long-range interactions on basis of electric and magnetic fields in a non-collisional plasma are considered by using a Particle-in-Cell (PIC) approach $^{16)}$. Furthermore, the (short-range) elastic and inelastic collisions between neutral and charged particles have to be considered. Therefore, the exchange in momentum and energy as well as chemical reactions is calculated by means of a Direct Simulation Monte Carlo (DSMC) method ${ }^{17)}$. A newly developed Fokker-Planck solver ${ }^{18)}$, also using PIC techniques in velocity space, numerically models the mid-range electron-electron and electron-ion Coulomb collisions.

It is expected that the coupling of these models, i.e., a PIC-DSMC-FP solver, allows for an accurate prediction of the thrust and the plasma properties of pulsed plasma thrusters in general, and particularly of SIMP-LEX / ADD SIMP-LEX.

\section{Conclusions and Outlook}

Several experimental studies investigating and improving the performance characteristics of the pulsed plasma thruster SIMP-LEX were reviewed. These studies aim at obtaining an optimum configuration for the application of a PPT on the satellite mission BW1.

Within the studies reviewed it was seen that an electrode configuration of $20 \mathrm{deg}$ flared, tongue shaped with small dimensions in width, length and inter-electrode gap was most beneficial. A further amelioration in the studies conducted at IRS found that optimizing the electrical circuit helped increase the total thrust efficiency by a factor of approximately $2^{13)}$, whereas the mean exhaust velocity was raised by nearly $40 \%$. Thus, the amount of propellant needed to fulfill the missions' needs is reduced tremendously. Although the system's mass of the thruster was increased by the heavier capacitors, the design change yields a higher payload ratio for the satellite.

At IRS, analytical models are applied to calculate the thruster's behavior in general (slug model) as well as in detail (numerical 3D code). For the first one, the change in inductance per plasma position was calculated using the 
Biot-Savart law. Calculations with the slug model were done to compare with the experimental results, and showed good accordance. However, due to a lack of a reliable prediction of the time-dependent plasma resistance and the assumptions taken into account, the model still has some potential for improvement ${ }^{6}$. Different solvers were developed to describe the effects occurring in the plasma of a PPT. The coupling of these solvers in a unifying numerical code will provide a powerful tool to predict performance and plasma characteristics, and, hence, save valuable experimental time.

Near future development at IRS will include performance characterization and electrical optimization of the thruster. A new test facility will be set up for life testing and eventually integration of the SIMP-LEX flight model. In the next step, the propellant feed system, electronic system and the telemetry for on-orbit health monitoring will be integrated for flight qualification. In order to provide data to verify the numerical code, several plasma diagnostic experiments are to be conducted comprising spectroscopy and interferometry. Coupled current and magnetic field measurements are scheduled to enable a comparison with the theoretical prediction using the Biot-Savart law. These experiments will be conducted at the University of Tokyo as part of a close research cooperation in the field of PPT development.

\section{Acknowledgments}

We would like to thank the German Aerospace Center (Deutsches Zentrum für Luft- und Raumfahrt; DLR) which supported this work under contract number FKZ-50-JR-0446.

Further, the experimental help of Mr. Bastian Steiner for the parametric investigation is well appreciated.

\section{References}

1) Laufer, R., Röser, H.-P. and the Lunar Mission BW1 Project Team: Lunar Mission BW1 - An Academic Low-Cost Small Lunar Exploration Satellite, 58 $8^{\text {th }} I A C$, IAC-07-A3.I.A.03, Hyderabad, India, 2007.

2) Bock, D., Schönherr, T., Herdrich, G., Lau, M. and Röser, H.-P.: Electric Propulsion Technology for Small Satellite Missions, $5^{\text {th }}$ ISPC, 42-181, Heraklion, Greece, 2008.

3) Nawaz, A.: Entwicklung und Charakterisierung eines gepulsten instationären MPD Triebwerks als Primärantrieb für Weltraumsonden, Dissertation, Universität Stuttgart, Germany, 2010.

4) Nawaz, A., Herdrich, G., Kurtz, H., Schönherr, T. and Auweter-Kurtz, M.: SIMP-LEX: Systematic Geometry Variation using Thrust Balance Measurements, $30^{\text {th }}$ IEPC, IEPC2007-168, Florence, Italy, 2007.

5) Nawaz, A., Bauder, U., Böhrk, H., Herdrich, G. and Auweter-Kurtz, M.: Electrostatic probe and camera measurements for modeling the iMPD SIMP-LEX, 43 ${ }^{\text {rd }}$ JPC, AIAA-2007-5280, Cincinnati, OH, USA, 2007.

6) Schönherr, T., Nawaz, A., Herdrich, G., Röser, H.-P. and Auweter-Kurtz, M.: Influence of electrode shape on performance of pulsed magnetoplasmadynamic thruster SIMP-LEX, Journal of Propulsion and Power, 25 (2009), pp. 380-386.

7) Nawaz, A., Lau, M., Herdrich, G. and Auweter-Kurtz, M.: Investigation of the Magnetic Field in a Pulsed Plasma Thruster, AIAA Journal, 46 (2008), pp. 2881-2889.
8) Koizumi, H., Noji, R., Komurasaki, K. and Arakawa, Y.: Plasma acceleration processes in an ablative plasma thruster, Physics of Plasmas, 14 (2007), pp. 033506-033506-10.

9) Palumbo, D. J. and Begun, M.: Plasma Acceleration in Pulsed Ablative Arc Discharges, Final Rept. Period 1974-1977, Fairchild Industries, New York, 1977.

10) Nawaz, A. and Auweter,-Kurtz, M.: Parametric Performance Characterization of Pulsed Plasma Thrusters, to be submitted to the IEEE Transactions on Plasma Science.

11) Antropov, N. N., Diakonov, G., Orlov, M., Popov, G. A., Tyutin, V. and Yakovlev, V.: Development and refinement of highly efficient $150 \mathrm{~J}$ APPT, $28^{\text {th }}$ IEPC, IEPC-03-061, Toulouse, France, 2003.

12) Lochner, R. H. and Matar, J. E.: Designing for Quality: An introduction to the best of Taguchi and Western methods of statistical experimental design, Quality Resources, White Plains, NY, USA, 1990.

13) Nawaz, A., Albertoni, R. and Auweter-Kurtz, M.: Thrust efficiency optimization of the pulsed plasma thruster SIMP-LEX, Acta Astronautica, 67 (2010), pp. 440-448.

14) Jahn, R. G.: Physics of Electric Propulsion, Dover Publications, Inc., Mineola, NY, USA, 2006.

15) Fertig, M., Petkow, D., Stindl, T., Auweter-Kurtz, M., Quandt, M., Munz, C.-D., Neudorfer, J., Roller, S., D'Andrea, D. and Schneider, R.: Hybrid Code Development for the Numerical Simulation of Instationary Magnetoplasmadynamic Thrusters, in High Performance Computing in Science and Engineering '08, Springer-Verlag, Berlin \& Heidelberg, Germany, pp. 585-597, 2009.

16) Munz, C.-D., Schneider, R., Sonnendrücker, E., Stein, E., Voß, U. and Westermann, T.: A finite-volume particle-in-cell method for the numerical treatment of the Maxwell-Lorentz equations on boundary-fitted meshes, Int. J. Numer. Meth. Engng., 44 (1999), pp. 461-487.

17) Petkow, D., Fertig, M., Herdrich, G. and Auweter-Kurtz, M.: Ionization Model within a 3D PIC-DSMC-FP Code, 39th AIAA Thermophysics Conference, AIAA-2007-4261, Miami, FL, USA, 2007.

18) D'Andrea, D., Munz, C.-D. and Schneider, R.: Modeling of electron-electron collisions for particle-in-cell simulations, FZKA 7218 Research Report, Forschungszentrum Karlsruhe - in der Helmholtz-Gemeinschaft, 2006. 